\title{
Supporting Task Shifting and Task Sharing in Nigeria: An Assessment of Family Planning Knowledge Retention of Community Pharmacists and Patent Proprietary Medicine Vendors
}

Sikiru Baruwa ( $\nabla$ sobaruwa@yahoo.com )

Population Council

Elizabeth Tobey

Population Council, Washington D.C

Emeka Okafor

Society for Family Health, Abuja

Kayode Afolabi

Federal Ministry of Health

Toyin O. Akomolafe

Population Council

Innocent Ubuane

Population Council

Jennifer Anyanti

Society for Family Health, Abuja

Aparna Jain

Population Council, Washington D.C

\section{Research Article}

Keywords: Task shifting and task sharing, Knowledge retention, Community Pharmacists, PPMVs, FP Jobs aids, FP knowledge, Injectable knowledge, implant knowledge

Posted Date: February 16th, 2022

DOI: https://doi.org/10.21203/rs.3.rs-1342368/v1

License: (1) This work is licensed under a Creative Commons Attribution 4.0 International License. Read Full License 


\section{Abstract}

Background: CPs and PPMVs are an important source of modern contraceptives in Nigeria, yet many lack the requisite knowledge and skills to capably provide these services. This skills gap might be addressed through targeted family planning training. This study measures family planning knowledge retention of CPs and PPMVs after receiving training in FP counseling and services in Kaduna and Lagos States, in Nigeria.

Methods: As part of the evaluation study, 559 CPs and PPMVs completed a self-administered questionnaire to assess their knowledge related to the provision of FP counseling, and injectable and implant contraceptive services at three points in time: 1) before the training; 2) immediately after the training; and 3) 9-months after the training. Multivariate logistic regression analysis was used to assess the effect of provider characteristics and receipt of job aids on FP knowledge retention 9 months after the training

Results: The study revealed the importance of jobs aids as influence on knowledge retention. CPs and PPMVs who reported having the Balanced Counseling Strategy plus (BCS+) counseling cards, were more likely to retain knowledge (AOR: $2.92 ; 95 \% \mathrm{Cl}$ : 1.01-8.40) at 9 months follow-up. Similarly, in terms of injectable knowledge, CPs and Tier 2 PPMVs who reported receiving the Medical Eligibility Criteria (MEC) Wheel were 2.1 times more likely to retain injectable knowledge 9-months later on (95\% Cl: 1.14-3.99).

Conclusion: CPs and PPMVs can be trained to offer FP counseling and injectable and implant services. Their knowledge can be supported through job aids, suggesting that they offer accurate information and quality services. For Nigeria, including Family planning job aids in the roll out of the task shifting and task sharing policy will be critical to a successful implementation.

\section{Introduction}

Despite many years of concerted efforts to improve family planning (FP) services and uptake by national governments and international organizations, unmet need for family planning remains high globally (1). Four of the six countries with the highest unmet need for FP are in SSA and include Democratic Republic of the Congo (DRC), Uganda, Nigeria, and Kenya (2). In Nigeria, 15\% of all women of reproductive age (1549) have an unmet need for FP (3). The current prevalence rate for modern contraceptive use in Nigeria is appropriately 12 percent (Nigeria Demographic and Health Survey (NDHS), 2019). (3) This translates to low levels of FP uptake and unwanted pregnancy and correlates with high maternal mortality ratio (4). Many women who need family planning are not using contraception" (3). Long-acting methods such as implants, and intrauterine devices (IUDs) are mainly available in public health centres while short acting reversible methods like emergency contraception, male condoms, and pills are primarily available from private chemists/patent medicine store. (3). Providing women with access to a full range of methods, including access to long-acting methods is important in ensuring they have the right to choose the method that is best suited to their needs. 
About $41 \%$ of women in Nigeria who use a modern contraceptive method receive their method from the private sector (3). Community pharmacists (CPs) and Patent Propriety Medicine Vendors (PPMVs) are popular sources of contraception among both public and private sources with about $22 \%$ of modern contraceptive users reported receiving their last method from a PPMV, and $12 \%$ from a community pharmacy (3). A CP is a trained pharmacist with full license to sell and buy prescription and nonprescription drugs. PPMVs are "persons without formal pharmacy training selling orthodox pharmaceutical products on a retail basis for profit" (5). In 2005, the number of PPMVs in Nigeria was estimated to be 200,000 , roughly 100 times greater than the number of registered pharmacies, and nearly four times the number of physicians (6). CPs and PPMVs are both currently regulated by the Pharmacists Council of Nigeria (PCN).

The main reasons why women reported use of the private sector include widespread availability, consistent drug stocks, extended hours, personable interactions, and no separate fees for consultations (7-8). However, CPs and PPMVs have not received formal training to provide FP services and often lack the requisite knowledge and skills to competently provide these services. Previous studies have shown that while many PPMVs provide FP services, they do not have the required knowledge to do so $(9-10)$.

\section{Task Shifting and Sharing Policy (TSTS)}

Based on the realization of severe health care worker shortage globally especially in sub-Sahara Africa, the World Health Organization (WHO) proposed task shifting to improve access to health care services (11). Task shifting involves shifting some tasks from one cadre to another, for example from doctors to nurses, midwives, or community health workers $(11$,$) . In Sub-Sahara Africa, the shortage of health work$ force is further heightened by a high burden of diseases; migration of trained health workers; lack of enabling work environment, and low staff morale (12 -13). The WHO provided global guidance on the process of TSTS implementation, which includes guidance on consultation, situation analysis, national endorsement, and regulatory framework. WHO guideline also specifies elements of quality assurance such as standardized training, supportive supervision, and certification and assessment, necessary to ensure quality of care (11).

To increase access to health services in Nigeria, the Federal Ministry of Health (FMOH) developed a TSTS Policy Guidelines in 2014 (14). The TSTS policy focuses on key priority areas such as Reproductive Health, Maternal and Child Health (RMNCH), family planning, HIV, tuberculosis, malaria and other communicable and non-communicable diseases in the essential health services package (14).

As part of the Federal Ministry of Health (FMoH) commitments to increasing access to high-quality FP services, efforts are being made to include CPs and PPMVs in the TSTS policy. The TSTS policy specifies that essential health care services of some family planning services can be shared with lower cadre health workers such as the Community Health Extension Workers (14). Regarding FP, task sharing is commonly practiced in the public health sector to community healthcare workers (15-16) but not implemented often in the private sector with CPs and PPMVs despite it being considered a high impact practice in FP (17). Literature has revealed that task shifting some of the family planning services to 
other cadres may be cost effective, increase access to and availability of maternal and reproductive health services without compromising standard of service delivery (18). However, task shifting can only be successful when supported by training, services restructuring, mentoring, supervision, and ongoing support from existing health system structures (19-21).

Training is a key strategy to build capacity of CPs and PPMVs to offer quality FP services, although some studies have shown that only about $30 \%$ of CPs and PPMVs received any training on FP counseling or provision of services $(22-23)$. Most trainings are targeted to public-sector providers (24) and not CPs and PPMVs. Where opportunities were available to train CPs and PPMVs, as business owners they were concerned about forgoing profits when participating in trainings. Trainings that address this barrier may better attract private sector participants and retain their participation throughout the training.

A systematic review (25) of the effect of FP trainings on service delivery in the public sector showed that FP trainings can positively influence provider knowledge, the quality of counseling and services rendered. One of these studies reviewed (26) found that FP training is positive in enhancing providers' average knowledge scores of side effects and warning signs for oral contraceptives and IUDs. They also found that providers not only increased their knowledge of contraception but also offered better quality of care to their clients. Another study in Nigeria reported a positive correlation between training and quality of FP counselling where trained nurses performed better in interpersonal relations, information giving, counseling, and mechanisms for encouraging continuity (27). A study in the Philippines found that a FP training intervention positively improved providers' knowledge and quality of care received by clients (28). In Ghana, a study found that trained counsellor provided more detailed and full information about all available contraception (29).

In the private sector, three studies have shown that FP trainings can improve provider knowledge and quality of services. A study in Gujarat, India, showed that after training in FP methods, private medical practitioners FP knowledge improved, and this resulted in better care for their clients (30). Another study in Nepal found positive improvements in client satisfaction at intervention clinics as compared to the control (31). In Nigeria, previous studies reveal that PPMVs' knowledge of injectable contraceptives increased with training (32 -33) and PPMVs' FP clients are also generally satisfied with the services received $(10,32)$. However, one study found no impact of training on provider behavior. A Study in Peru that assessed the effect of quality-of-care improvements after provider training on the job aids, found weak connection between training providers on FP job aids training and client's outcomes (34).

Our study builds on this body of literature and explores the effect of FP trainings on CPs and PPMVs knowledge retention. The study also looks at the role of job aids in supporting knowledge retention over time.

\section{Methods}

\section{Data}


Data for this analysis come from an evaluation of the IntegratE Project, a pilot project that seeks to improve the quality of FP services delivered by CPs and PPMVs through a tiered accreditation system. Beginning in January 2019, 1,465 CPs and PPMVs located in Kaduna and Lagos States were trained by the IntegratE project. As part of the evaluation study, 559 CPs and PPMVs completed a self-administered questionnaire to assess their knowledge related to the provision of FP counseling, and injectable and implant contraceptive services depending on their tier at three points in time: 1) before the training; 2) post/immediately after the training; and 3) 9-months after the training. The assessments were developed from established knowledge assessment tests used by the Project's training partners.

All questionnaires asked the same knowledge questions on FP counselling, injectable and implants contraceptives. The pre-test assessment also included questions on respondent characteristics and their cadre, state, experience providing injectable and implant contraceptives. The nine-month questionnaire included questions on services offered by CPs and PPMVs since the training, and their experience with the intervention including training received, job aids and monitoring visits. The results from the pre and posttest and 9 months follow up questionnaires were compared and analyzed in this paper.

All participants enrolled in the study were given detailed information about the study before consenting to participate. The study protocol was submitted and approved by the Population Council Institutional Review Board.

\section{The IntegratE Project}

The IntegratE Project, supported by Bill \&Melinda Gates foundation and MSD for mothers was implemented by a consortium of partners led by Society for Family Health in Nigeria. Other partners include Marie Stopes International of Nigeria, PharmAccess Foundation, Planned Parenthood Federation of Nigeria, and the Population Council. The project adopted a tiered accreditation approach where providers were categorized into three tiers based on their health qualifications. Each tier was authorized to practice FP services consistent with their qualifications (see Table 1).

All providers were trained in FP counseling using the balanced counseling strategy plus (BCS+) toolkit. Sessions focused on (a) different contraceptive methods available in Nigeria; (b) instruction and practice sessions to offer client-centered FP counseling using BCS+; and (c) instruction on how and when to refer clients for FP to other sources of care based on client's medical eligibility.

In addition to FP counselling training, CPs and Tier 2 and Tier 3 PPMVs received further training on administration of injectable contraceptives and administration and removal of implant contraceptives. Providers practiced inserting implants on models and inserting injectables and implants on clients who voluntarily choose either of these two methods. CPs and PPMVs were referred to the social marketing sector to procure additional commodities. Supervisory monitoring visits were conducted within three months of the training to gauge compliance with protocols related to FP and infection prevention and control. 
After the FP training, CPs and PPMVs were given the BCS+ toolkit, which includes: a) BCS+ counseling cards; b) BCS+ algorithm; c) FP flip chart; and d) FP method brochures. In addition to the BCS+ toolkit, Tier 2 and Tier 3 PPMVs and CPs were given the Contraceptive Medical Eligibility Criteria (MEC) wheel check. An explanation of each tool is available in Table 2.

\section{Variables}

\section{Dependent variables}

Three dependent variables were used in this paper: 1) general FP knowledge retention, 2) injectable knowledge retention, and 3) implant knowledge retention. General FP knowledge retention was assessed among Tier 1 and Tier 2 PPMVs and CPs, while injectable and implant knowledge retention were assessed only among Tier 2 PPMVs and CPs, as these tiers were trained in the provision of these methods. General knowledge and injectable knowledge categories each included 11 items, while 7 items were included in implant knowledge. Knowledge retention was measured for each item from posttest to the 9-month follow-up survey. Those who correctly answered a knowledge assessment question at posttest and answered it correctly at 9 months had the knowledge after the training and retained it over time. Respondents who did not know the item at posttest or at 9 months, who did not know it at posttest but did at 9 months, and who did know it at posttest and at 9 months were not considered to have retained knowledge from posttest to 9-months.

Overall summary knowledge retention scores were developed for general FP knowledge, injectable knowledge, and implant knowledge. Scores were created for each of the three outcome variables separately. The total number of items that each respondent retained from posttest to 9 months was added. For general and injectable knowledge this is 11 items and for implant knowledge this is 7 items. Retention includes those who answered the question correctly at posttest and 9 months, while all other combinations are not counted as retention. For each outcome variable, the mean number of items that were retained was calculated. Those who scored above the mean were coded as 1 for high knowledge retention, and those who scored below the mean were coded as 0 for low knowledge retention. The mean scores were 5.7 out of 11 for general knowledge, 7.9 out of 11 for injectable knowledge, and 2.7 out of 7 for implant knowledge. Those who scored 6 or higher for general, 8 or higher for injectable, and 3 or higher for implant were considered to have high knowledge retention.

\section{Independent variables}

The key independent variable for this analysis were the five job aids given to all providers trained by the project. The job aids were categorized into three groups for their similarities: category 1 included BCS+, BCS+ algorithm, or FP flip charts (an alternative to BCS+), category 2 were the FP method brochures, and category 3 was the MEC wheel. For each of the three categories of job aids, responses were categorized as those who received the job aid during or after the training and those who did not receive the job aid. 
Additional covariables considered were gender, age, tier, state, previous health facility experience, and receipt of specific job aids. Education was also considered but because it was used in the determination of tiers, it was highly correlation with tier and excluded from the analysis. For implant and injectable knowledge, a variable on whether the provider had offered these specific services in the 30 days preceding the interview was also included.

\section{Data analysis}

Descriptive statistics were calculated for respondent characteristics at pretest and are presented by tier. Descriptive statistics for knowledge retention for general FP (among all providers), injectable (among Tier 2 PPMVs and CPs), and implant (among Tier 2 PPMVs and CPs) were conducted. The proportion who correctly answered each item at posttest was calculated, and among those who answered correctly at posttest, the proportion that retained the knowledge at 9 months is presented. The denominator for each item is dependent on the proportion who answered correctly at posttest.

Three multivariate logistic regression models were used to assess the effect of provider characteristics and receipt of job aids on general FP knowledge retention, injectable knowledge retention, and implant knowledge retention 9 months after the training. The outcome variable in each of the three models was high versus low knowledge retention according to the mean scores (as described above). The model for general knowledge was conducted among all respondents, while the implant and injectable models were conducted among Tier 2 PPMVs and CPs.

\section{Results}

Table 3 presents background characteristics of providers included in the evaluation study by total and provider type. These data were collected before the FP training began. More females $(60.3 \%)$ than males (39.2\%) were included in the study. This was the case across all provider types. Most providers were between 30-49 years old (63.4\%), and Tier 2 PPMVs were slightly younger than CPs and Tier 1 PPMVs. While a nearly even split of respondents came from Lagos and Kaduna overall, more CPs were enrolled in Lagos (72.8\%) and more Tier 2 PPMVs were enrolled in Kaduna (93.0\%). CPs (91.9\%) and Tier 2 PPMVs (85.0\%) were more likely to have ever worked in a health facility compared to Tier 1 PPMVs (45.9\%). Slightly more than half $(51.0 \%)$ of providers had never received any structured FP training and most who did, received it over a year before the intervention ago (35.4\%).

Table 4 shows retention of general FP counseling knowledge at posttest among all providers, and at 9months follow-up but only for those providers who had the knowledge at posttest to assess knowledge retention. For many indicators, provider knowledge was high at posttest and among those who responded correctly to the question at posttest, many were able to retain the information. For example, $98 \%$ of all providers correctly reported that condoms protect against pregnancy and HIV. Of those $98 \%$, nearly all $99.5 \%$ continued to retain this information. Similarly, many providers $(89.6 \%)$ at posttest knew the Intrauterine Device (IUD) was a modern method and most of them (87.4\%) retained this information. In several instances, for example whether clients should be allowed to handle a sample of FP method 
during counseling, posttest knowledge was a little more than half (55.8\%). But nearly all of those who had this knowledge at posttest retained it 9-months later (94.2\%).

For other knowledge measures, however, initial knowledge after the training was low and many of those who had the knowledge posttest lost it 9-months later. Only $27.0 \%$ knew that lactational amenorrhea method (LAM) is a modern method at posttest, and among them, only $18.5 \%$ retained this knowledge 9months later. This was also the case for standard days method (SDM).

Table 5 shows knowledge retention of injectable contraceptive information including both Depo-Provera and Sayana Press. These questions were only asked among those trained in administration of injectable services - CPs and Tier 2 PPMVs. Overall knowledge was high for nine out of eleven questions at posttest, ranging from a low of $79.4 \%$ for knowledge of where Depo-Provera is injected to a high of $94.8 \%$ for the frequency of Depo-Provera injections. Retention of these nine questions remained for most who knew this information as posttest. Fewer had correct knowledge at posttest for two questions - the location of the Sayana Press injection (53.6\%) and disposal of sharp box contents (42.3\%). Most of those who knew where to dispose sharps at posttest retained the knowledge 9-months later $(82.4 \%)$ while about half still knew where Sayana Press injection was given on the body 9-months later (51.9\%).

Table 6 shows retention of implant knowledge 9-months after the posttest for CPs and Tier 2 PPMVs. Across the seven questions, knowledge was high at posttest for four questions and was retained by most providers 9-months later. Several indicators were less known at posttest including the angle to insert the Implanon NXT needle (46.4\%) and that implants may be difficult to remove when inserted under the fat $(61.2 \%)$. For these two questions retention was lower than the other questions at $33.3 \%$ and $67.0 \%$, respectively.

Multivariate models are presented in Table 7 for all knowledge retention areas. For FP knowledge retention, providers who reported having the BCS+ counseling cards, BCS+ algorithm or the FP flip chart were more likely to retain knowledge on six or more questions (AOR: $2.92 ; 95 \% \mathrm{Cl}: 1.01-8.40)$. CPs and those who ever worked in a health facility were also more likely to retain knowledge 9 -months later on six of the eleven knowledge questions.

In terms of injectable knowledge, CPs and Tier 2 PPMVs who reported receiving the MEC Wheel were 2.13 times more likely to retain injectable knowledge 9-months later on at least 6 injectable knowledge questions (95\% Cl: 1.14-3.99). The odds of retaining injectable information were also more likely for females compared to males and for those who ever worked in health facility. Similar results were observed for implant knowledge retention except for ever worked in a health facility which was not statistically significant.

\section{Discussion}

This study is one of the few studies of FP trainings to focus on both CPs and PPMVs' FP knowledge retention. Unlike recent studies that examine knowledge retention with injectables training, this study 
also considers knowledge retention of implants. This study showed that nine months after the FP training, knowledge of key general FP, injectable and implant questions was retained. This suggests that CPs and PPMVs can be trained to provide a wide array of FP services. The findings further demonstrate that targeted training of CPs and PPMVs enhances FP knowledge. Results from this study are consistent with other studies in Nigeria that found positive impact of training on providers' knowledge (10, 27,). This finding is promising for the strategy of task shifting task sharing in resource limited settings by expanding the role of CPs and PPMVs in FP service delivery in view of the major shortage of skilled providers for delivering FP services, especially for injectables and LARCs.

Another important finding from this study is that certain FP knowledge questions were better retained by CPs and PPMVs than other knowledge questions. For example, implant knowledge decreased 9 months later for two questions: the angle to insert the Implanon NXT needle and that an implant may be difficult to remove when inserted under the fat. Also, while this study focused on knowledge retention of providers who had the knowledge at posttest, it is important to note there were CPs and PPMVs who gained the information after posttest, suggesting that learning can be achieved through other implementation activities like monitoring and supervisory visits.

Overall, the results of this study showed that CPs and PPMVs retained injectable knowledge better than implant knowledge. Prior to the training, many of the CPs and PPMVs had already been providing injectables (33) without participating in any trainings or included in a supportive environment and even at posttest, the injectable knowledge scores were better than the implant knowledge scores. After trainings, providers have been offering injectable services more frequently than implant services; routine service data (35) showed that about $30 \%$ and $7 \%$ of women received injectables and implants, respectively. So, administering injectables over time may support providers' retention of knowledge. To ensure method choice, however, additional support to providers is necessary so that they are offering full and complete information around implants and other FP methods. Results also suggest that trainings should focus on how and where to dispose sharps.

The study showed the importance of jobs aids for post training knowledge reinforcement for the providers. CPs and PPMVs who reported receiving job aids were more likely to answer the key knowledge questions correctly. This finding suggests that providing jobs aids to support CPs and PPMVs FP service provision can be effective in retaining knowledge over time. A similar study in Nigeria has shown that in addition to training, providing FP job aids was another strategy that can help PPMVs in effectively providing FP services over time (36). FP Job aids supported healthcare workers with procedural, informational, or decisional "need-to-know" information in a simplified way (36). Many studies in Africa have reported the usefulness of FP job aids. In Uganda and the Democratic Republic of Congo, (37) findings show that about half of the providers surveyed reported using FP checklists between 7 and 24 months after training. The providers who used these job aids indicated that they found them very useful. Another study conducted in South Africa reveals that a reinjection screening checklist aided the providers to excludes pregnancy for late arriving depot-medroxyprogesterone acetate (DMPA) clients and ultimately prevented unintentional discontinuation among those clients (38). However, this study reported no 
difference in terms of clients' timeliness for reinjections. The multivariate results showed that among the FP jobs aids, the BCS cards and FP methods brochures helped CPS and PPMVs retained general FP, injectable and implants knowledge nine months after training. The CPs and PPMVs may likely or tend to use more the BCS cards and FP methods brochures in their day-to-day interactions with their clients. Providing FP jobs aids is one thing using the jobs aids is another thing. CPs and PPMVs must be trained on the use of jobs aids to reinforce their FP knowledge and enhance quality FP counselling and services.

This study also demonstrated that provider cadre was a significant factor in FP knowledge retention. General FP knowledge retention was more likely among CPs compared to PPMVs. Implant knowledge retention was more likely among CPs compared to PPMVs. This may be due to the fact the CPs have more years of prior training and exposure to health education and theoretical information in schools. Employing observational studies to assess the CPs and PPMVs FP competency and quality of care received by their clients may be a better way to compare these two providers.

We also found that having ever worked in health facilities was positively associated with retention of knowledge. General FP knowledge retention was more likely among CPs/PPMVs that have ever worked in a health facility compared to those that have not. Also, injectable knowledge retention was more likely among CPs/PPMVs that have ever worked in a health facility compared to those that have not. This result may be due to CPs and PPMVs exposure to training and practice in the public or private health facilities. CPs and PPMVs that have worked in health facilities were more likely to retain FP knowledge although this was not found statistically significant in a similar PPMV's study in Nigeria (34).

Provision of an injectable and implant methods in the past 30 days was not found to be a statistically significant predictor of knowledge. Administering injection and implant within 1 month not sufficient to maintain knowledge among CPs and PPMVs. However, we need more studies that we look at the effect of client load or repeated practice on knowledge retention after training. Further, age, years of work and education attainment may not enhance retention of knowledge However, training program for CPs and PPMVs may be made more effective by taking into consideration gender, cadre, ever worked at health facilities.

\section{Limitations}

There are a number of limitations to this study. The first was that our questions about FP knowledge only focused on general FP counselling, injectables and implants knowledge. We did not examine the CPs and PPMVs knowledge on FP methods side effect. The results may not reflect the comprehensive CPs and PPMVs knowledge of FP.

The second limitation is that COVID affected how data were collected in the follow-up interview where no in-person contact was allowed. In the wake of the COVID-19 pandemic research assistants administered the questionnaire over the phone. They read questions and responses to CPs and PPMVs before recording their responses on the Android tablet. This may have inherent flaws especially in the recording of responses and network problems. However, the research assistants were properly trained to ensure 
they had the necessary skills required for this data collection activity. Also, we endeavored to backcheck to validate the responses with randomly selected CPs and PPMVs during the data collection.

\section{Conclusion}

The study has showed the likely impact of training on knowledge retention. The findings revealed that targeted training helped CPs and PPMVs retain the knowledge they were exposed to during training intervention. We can also conclude that apart from training, jobs aids were necessary for post training reinforcement and help CPs and PPMVs knowledge retention over time. Interventions need to take into consideration provision and training on FP job aids as one key strategy to help the CPs and PPMVs in providing FP services over time

\section{Abbreviations}

CP: Community Pharmacist; FP: Family planning; PPMV: Patent and proprietary medicine vendor; MEC: Medical Eligibility Criteria; BCS+: Balanced Counselling Strategy plus; OR: Odds ratio; PCN: Pharmacists Council of Nigeria; TSTS: Task-shifting and task-sharing.

\section{Declarations}

\section{Acknowledgements}

The study was conducted in collaboration with the Federal Ministry of Health of Nigeria, Pharmacists Council of Nigeria, National Association of Patent and Proprietary Medicine Dealers, State Ministries of Health in Kaduna and Lagos and the IntegratE consortium partners Society for Family Health, Marie Stopes International, Planned Parenthood Federation of Nigeria, and PharmAccess. We thank Adebola Adedimeji at the Population Council who reviewed this manuscript. The successful completion of these studies would not have been possible without the dedication of the research team and data collectors. We further acknowledge all the CPs and PPMVs who volunteered their time and provided valuable information during this study.

\section{Author's contributions}

SB managed the overall preparation, participated in the conceptualization, and prepared the first draft of the manuscript. ET participated in the conceptualization, conducted the analysis, and drafted the methodology section. UO and TA managed the coordination of data collection and data management and reviewed the manuscript. EO led the funding acquisition for the Integrate project, including the study, and reviewed drafts of the manuscript. KA and JA reviewed and edited the manuscript. AJ was the principal investigator and led the development of the overall study's methodology, guided the conceptualization and analysis, and drafted the results section and reviewed the manuscript. All authors have read and approved this version of the manuscript. 


\section{Funding}

Bill \& Melinda Gates Foundation [INV-007278 Formerly OPP1173898] and MFM-19-17114. The research in this publication was supported by funding from The Bill \& Melinda Gates Foundation [INV-007278 Formerly OPP1173898] and MSD through its MSD for Mothers program (MFM-19-17114) and is the sole responsibility of the authors. MSD for Mothers is an initiative of Merck \& Co., Inc., Kenilworth, NJ, U.S.A.

\section{Availability of data and materials}

The dataset analyzed during the current study will be available on the Population Council's Dataverse, https://dataverse.harvard.edu/dataverse/popcouncil. They will also be available from the corresponding author on reasonable request.

\section{Ethics approval and consent to participate}

The research protocol received ethical approval from the Population Council's Institutional Review Board (Protocol 878), Nigeria's National Health Research Ethic Committee. All methods were performed in accordance with the relevant guidelines and regulations stated in the approved protocol. Verbal informed consent was received before the beginning enrollment and follow-up interview.

\section{Consent for publication}

Not applicable.

\section{Competing interests}

The authors declare that they do not have any competing interests. The funders of this study did not have any role in the design, planning, or execution of this work.

\section{References}

1. Darroch JE, Sedgh G, Ball H. Contraceptive technologies: responding to women's needs. New York: Guttmacher Institute. 2011;201(1):1-51.

2. Cleland J, Shah IH, Benova L. A fresh look at the level of unmet need for family planning in the postpartum period, its causes and program implications. Int Perspect Sex Reprod Health. 2015;41(3):155-62.

3. National Population Commission (NPC) [Nigeria] and ICF. 2019. Nigeria Demographic and Health Survey 2018. Abuja, Nigeria, and Rockville, Maryland, USA: NPC and ICF.

4. United Nations, Department of Economic and Social Affairs. World Family Planning; New York: UN. 2017

5. Brieger WR, Osamor PE, Salami KK, Oladepo O, Otusanya SA. Interactions between patent medicine vendors and customers in urban and rural Nigeria. Health Policy Plan. 2004 May;19(3):177-82. 
6. Bames J, Chandani T, Feeley R. Nigeria Private Sector Health Assessment-Private sector partnership one project; 2008

7. Brugha R, Zwi A (2002) "Improving the quality of private sector delivering of public health services: challenges and strategies." Health Policy Plan, 13:103-120.

8. Adetunji JA (1991) "Response of parents to five killer diseases among children in a Yoruba community, Nigeria." Social Science Medicine 32:1379-1387

9. Ajuwon AJ, Oshiname FO, Imaledo J, Ajayi O, Dipeolu IO. Delivery and utilisation of injectable contraceptive services in rural Nigeria: learnings from the perspectives of patent medicine vendors and women of reproductive age. Afr J Biomed Res 2016; 19:79-87

10. Ujuju C, Adebayo SB, Anyanti J, Oluigbo O, Muhammad F, Ankomah A. (2014). An assessment of the quality of advice provided by patent medicine vendors to users of oral contraceptive pills in urban Nigeria. Journal of Multidisciplinary Healthcare. Volume 7

11. World Health Organization. Task shifting: Global recommendations and guidelines. Geneva: World Health Organization; 2008.

12. Callaghan M, Ford N, Schneider H. A systematic review of task-shifting for HIV treatment and care in Africa. Hum Resour Health. 2010;8:8. http://dx.doi. org/10.1186/1478-4491-8-8

13. Lehmann U, Van Damme W, Barten F, Sanders D. Task shifting: The answer to the human resources crisis in Africa? Hum Resour Health. 2009;7:49. http://dx.doi. org/10.1186/1478-4491-7-49

14. World Health Organization (WHO) (2007) Task Shifting to Tackle Health Worker Shortages. WHO, Geneva.

15. Federal Ministry of Health Task-shifting and task sharing policy for essential health care services in Nigeria. 2014.

16. World Health Organization (2017): Task sharing to improve access to Family Planning/Contraception. Summary Brief. Geneva, Switzerland. https://apps.who.int/iris/bitstream/handle/10665/259633/WHO-RHR-17.20eng.pdf;jsessionid=9D05F66331F909F3693312AFB3D11C80? sequence $=1$

17. Stanback J, Spieler J, Shah I, Finger WR. 2010. Community-based health workers can safely and effectively administer injectable contraceptives: conclusions from a technical consultation. Journal of Contraception. 81: 181-184 (2010).

18. World Health Organization, U.S. Agency for International Development, Family Health International (FHI). Community-Based Health Workers Can Safely and Effectively Administer Injectable Contraceptives: Conclusions from a Technical Consultation. Research Triangle Park (NC): FHl; 2010.

19. Dawson, A. J, Buchan, J., Duffield, C., Homer, C. S. E \&Wijewardena, K. (2013). Task shifting and sharing in maternal and reproductive health in lowincome countries: a narrative synthesis of current evidence Journal of Health Policy and Planning 2014;29:396-408.

20. Fairall L, Bachmann MO, Lombard C, Timmerman V, Uebel K, Zwarenstein M, et al. Task shifting of antiretroviral treatment from doctors to primary-care nurses in South Africa (STRETCH): A pragmatic, 
parallel, cluster-randomised trial. Lancet. 2012;380:889-898. http://dx.doi.org/10.1016/S0140-

6736(12)60730-2

21. Emdin C, Millson P. A systematic review evaluating the impact of task shifting on access to antiretroviral therapy in Sub-Saharan Africa. Afr Health Sci. 2012;12(3):318-324. http://dx.doi.org/10.4314/ahs.v12i3.11

22. Callaghan M, Ford N, Schneider H. A systematic review of task-shifting for HIV treatment and care in Africa. Hum Resour Health. 2010;8:8. http://dx.doi. org/10.1186/1478-4491-8-820.

23. Drug Shops and Pharmacies: Sources for family planning commodities and information. Washington, DC: USAID; 2013 Jun. Available from http://www.fphighimpactpractices.org/briefs/drug-shops-and-pharmacies

24. O. A. Ajuwon, Ademola, John Imaledo, Frederick Oshiname, "Research and Recommendations on the Delivery of Injectable Contraceptive Services by Patent Medicine Vendors in Rural Nigeria," Res. Triangle Park. NC FHI 360, 2013.

25. A. I. and A. O. Babatunde Ahonsi, Ishaku Salisu, "Providers' and key opinion leaders' attitudes, beliefs and practices regarding emergency contraception in Nigeria," Popul. Counc. Inc, 2012

26. RamaRao $S$ and Mohanam R, The quality of family planning programs: concepts, measurements, interventions, and effects, Studies in Family Planning, 2003, 34(4):227-248.

27. Costello $\mathrm{M}$ et al., A client-centered approach to family planning: the Davao project, Studies in Family Planning, 2001, 32(4):302-314

28. Kim Y-M et al., Improving the quality of service delivery in Nigeria, Studies in Family Planning, 1992, 23(2):118-127.

29. Jain AK et al., Evaluation of an intervention to improve quality of care in family planning program in the Philippines, Journal of Biosocial Science, 2012, 44(1):27-41

30. Huntington D, Lettenmaier $C$ and Obeng-Quaidoo I, User's perspective of counseling training in Ghana: the "mystery client" trial, Studies in Family Planning, 1990, 21(3):171-177.

31. Agha $S$ et al., A Quasi-Experimental Study to Assess the Performance of a Reproductive Health Franchise in Nepal, Washington, DC: Abt Associates Inc., 2003

32. Ishaku SM, Afolabi K, Chace Dwyer S, Okunade F, Uzomba C, Adebayo A, Tobey E, Jain A. 2018. Examining and Strengthening the Provision of Injectable Contraception in Nigeria. Research Report. Washington, DC: Population Council, The Evidence Project.

33. Chace Dwyer S, Ishaku SM, Okunade F, Reichenbach L, Jain A. 2018. Feasibility of patent and proprietary medicine vendor provision of injectable contraceptives: preliminary results from implementation science research in Oyo and Nasarawa, Nigeria. Contraception 98 (2018) 460-462.

34. Nguyen DTK, Leung KK, McIntyre L, Ghali WA, Sauve R. Does integrated Management of Childhood Illness (IMCl) training improve the skills of health workers? A systematic review and meta-analysis. PLoS One. 2013;8(6):e66030. doi: 10.1371/journal.pone.0066030. 
35. Barge S, Patel BC and Khan I, Use of private practitioners for promoting oral contraceptive pills in Gujarat, Working Paper, Vadodara, Gujarat, India: Center for Operations Research \& Training, 1995, No. 5. Agency for International Development (USAID)/Commercial Market Strategies Project, 2002.

36. DHIS2 Community Pharmacies and PPMVs Family Planning Instance https://fp.dhis2nigeria.org.ng/dhis/dhis-web-dashboard/

37. Knebel E, Lundahl S, Raj AE, Abdallah H, Ashton J, Wilson N. "The use of manual job aids by health care providers: what do we know?" Issue paper, the quality assurance project; 2000.

38. Tumlinson K, Hubacher D, Wesson J, Lasway C. Measuring the usefulness of family planning job aids following distribution at training workshops. J Biosoc Sci. 2010;42(5):695-8

39. Baumgartner JN, Morroni C, Mlobeli RD, Otterness C, Buga G, Chen M. Impact of a provider job aid intervention on injectable contraceptive continuation in South Africa. Stud Fam Plann. 2012;43:305314

\section{Tables}

Table 1: Tiered Accreditation Training 
Tier 1 PPMVs (i.e. PPMVs lacking health qualifications and any training
1. Ability to read and write.

2. Attainment of 21 years of age.

3. Qualified personnel will include those who attempted to obtain a First School Leaving Certificate.

4. Also, in this category are Bachelor's degree in biological sciences or any other disciplines (outside of health related disciplines
1. Sell OTC and manage common illnesses.

2. FP services will include condoms, cycle beads, emergency contraceptives, refill of pills but not initiation, counselling, and referrals.

3. However, persons who have training on the use of malaria rapid diagnostic test-kits (m RDT) may use the test kits to test patients for malaria.
Tier 2 PPMVs (i.e. Health Qualified PPMVs):
1. Must fulfil the tier 1 eligibility criteria.

2. In addition, must possess degree in Health discipline as recognized by the Pharmacists Council of Nigeria

3. They could be Nurses, Midwives, CHEWs, CHOs
1. Provide select PHC services in line with the Task shifting and Task sharing policy.

2. Provide tier 1 services as well as use Rapid Diagnostic Test kits (RDTs), administer amoxicillin DT, conduct HIV Self Testing, sell self-injecting contraceptives and refer patients to PHCs and higher level facilities for nutrition counselling and treatment of any other common ailment.

3. They will also be allowed to initiate and administer LARC particularly implants if they have received training on comprehensive FP services in line with the Task Shifting and Task sharing policy.
Tier 3 PPMVs (i.e. Health Qualified PPMVs
1. These persons must fulfill the tier 1 eligibility criteria.

2. Must be Pharmacy technician
1. PPMV operators in this tier will be enabled to provide selected PHC services (in line with the Task shifting and Task sharing policy)

2. They will provide tier 1 services as well as use Rapid Diagnostic Test kits (RDTs), administer amoxicillin DT, conduct HIV Self Testing if trained

3. Sell self-injecting contraceptives and refer patients to PHCs and higher level facilities for nutrition counselling and treatment of any other common ailment 


\begin{tabular}{|c|c|c|}
\hline $\begin{array}{l}\text { Type of } \\
\text { Job Aid }\end{array}$ & Description & $\begin{array}{l}\text { Providers } \\
\text { who } \\
\text { received } \\
\text { job aids }\end{array}$ \\
\hline $\begin{array}{l}\text { BCS+ } \\
\text { counseling } \\
\text { cards }\end{array}$ & $\begin{array}{l}\text { It consists of information on pregnancy checklist, FP methods (use, side } \\
\text { effects and duration), and information on HIV risk assessment, } \\
\text { counseling, and testing }\end{array}$ & $\begin{array}{l}\text { All } \\
\text { providers }\end{array}$ \\
\hline $\begin{array}{l}\text { BCS+ } \\
\text { Algorithm }\end{array}$ & $\begin{array}{l}\text { Algorithm provides steps and questions to ask in the Pre-Choice Stage, } \\
\text { Method Choice Stage, Post-Choice Stage. It also offers systematic } \\
\text { screening of STIs / HIV including Prevention, Risk Assessment, and } \\
\text { Counseling and Testing Stage. }\end{array}$ & $\begin{array}{l}\text { All } \\
\text { providers }\end{array}$ \\
\hline $\begin{array}{l}\text { FP Flip } \\
\text { Chart }\end{array}$ & $\begin{array}{l}\text { The FP Flip Chart contains information about FP methods (short-term, } \\
\text { long-term, and permanent use), information on advantages and } \\
\text { disadvantages for each method, possible side effects for each method, } \\
\text { how to conduct counseling, HIV information, and pregnancy checklist. }\end{array}$ & $\begin{array}{l}\text { All } \\
\text { providers }\end{array}$ \\
\hline $\begin{array}{l}\text { FP method } \\
\text { brochures }\end{array}$ & $\begin{array}{l}\text { Pamphlets and leaflets on methods including their effectiveness, general } \\
\text { information, how method works, important facts, side effects, how to use, } \\
\text { and next steps. }\end{array}$ & $\begin{array}{l}\text { All } \\
\text { providers }\end{array}$ \\
\hline $\begin{array}{l}\text { MEC } \\
\text { Wheel } \\
\text { Check list }\end{array}$ & $\begin{array}{l}\text { This wheel contains the medical eligibility criteria for starting use of } \\
\text { contraceptive methods. } \\
\text { It guides family planning providers in recommending safe and effective } \\
\text { contraception methods for women with medical conditions or medically- } \\
\text { relevant. characteristics. The wheel includes recommendations on } \\
\text { initiating use of nine common types of contraceptive methods. }\end{array}$ & $\begin{array}{l}\text { Tier } 2 \\
\text { PPMVs, } \\
\text { Tier } 3 \\
\text { PPMVs } \\
\text { and CPs }\end{array}$ \\
\hline
\end{tabular}

Table 3. Background Characteristics of Private Providers in IntegratE Project Evaluation by Total and Provider Type, at Pretest 


\begin{tabular}{|c|}
\hline $\begin{array}{l}\text { CPs } \\
(n=272)\end{array}$ \\
\hline
\end{tabular}

\section{Gender of Respondent}

\begin{tabular}{|c|c|c|c|c|}
\hline Male & 47.8 & 30.0 & 33.8 & 39.2 \\
\hline Female & 52.2 & 70.0 & 62.2 & 60.3 \\
\hline \multicolumn{5}{|l|}{ Respondent Age } \\
\hline $21-29$ & 11.8 & 19.2 & 12.2 & 14.7 \\
\hline $30-39$ & 33.5 & 43.7 & 28.4 & 36.7 \\
\hline $40-49$ & 25.7 & 21.6 & 44.6 & 26.7 \\
\hline $50+$ & 24.6 & 14.1 & 14.9 & 19.3 \\
\hline \multicolumn{5}{|l|}{ State } \\
\hline Lagos & 72.8 & 7.0 & 51.4 & 44.9 \\
\hline Kaduna & 27.2 & 93.0 & 48.6 & 55.1 \\
\hline \multicolumn{5}{|l|}{$\begin{array}{l}\text { Ever worked in a health } \\
\text { facility }\end{array}$} \\
\hline No & 4.8 & 15.0 & 50.0 & 14.7 \\
\hline Yes & 91.9 & 85.0 & 45.9 & 83.2 \\
\hline \multicolumn{5}{|c|}{ Last structured FP training } \\
\hline Never & 54.0 & 47.9 & 48.6 & 51.0 \\
\hline Within the past month & 0.4 & 0.9 & 4.1 & 1.1 \\
\hline 2-6 months ago & 2.6 & 4.7 & 12.2 & 4.7 \\
\hline 7-12 months ago & 4.0 & 7.0 & 2.7 & 5.0 \\
\hline Over 1 year ago & 34.2 & 38.5 & 31.1 & 35.4 \\
\hline
\end{tabular}

Table 4: Knowledge retention of FP from posttest to 9 months among CPs, Tier 1 PPMVs and Tier 2 PPMVs $(n=559)$ 
Posttest 9-months later, among those that answered

$(n=559) \quad$ correctly at posttest

Method that prevents both pregnancy and HIV

$(n=548)$

OCPs, implant, ECP (incorrect)

$2.0 \quad 0.5$

Condoms (correct)

98.0

99.5

ECPs prevent pregnancy when taken up to 7 days

$(n=323)$

Incorrect

40.6

13.0

Correct

57.8

87.0

$(n=137)$

SDM is a modern method

Incorrect

75.5

75.9

Correct

24.5

24.1

$(n=151)$

LAM is a modern method

Incorrect

73.0

81.5

Correct

27.0

18.5

$(n=501)$

IUD is a modern method

Incorrect

10.4

12.6

Correct

89.6

87.4

$(n=502)$

Periodic abstinence is not a modern method

Incorrect

10.2

4.2

Correct

89.8

95.8

$(n=270)$

Copper IUD is not a hormonal method

Incorrect

51.7

48.5

Correct

48.3

51.5

A woman seeking FP selects her contraceptive method

$(n=486)$ 
Posttest 9-months later, among

those that answered

$(n=559) \quad$ correctly at posttest

\begin{tabular}{lll}
\hline Incorrect & 13.1 & 6.8 \\
\hline Correct & 86.9 & 93.2 \\
\hline $\begin{array}{l}\text { Clients should be allowed to handle a sample of FP method } \\
\text { during counseling }\end{array}$ & & $(\mathrm{n}=312)$ \\
\hline $\begin{array}{l}\text { Incorrect } \\
\text { Correct }\end{array}$ & 44.2 & 5.8 \\
\hline $\begin{array}{l}\text { Clients should not be asked if over } \mathbf{1 8} \text { years to assist them in } \\
\text { choosing a method }\end{array}$ & 55.8 & 94.2 \\
\hline $\begin{array}{l}\text { Incorrect } \\
\text { Correct }\end{array}$ & 25.8 & $1 \mathrm{n}=405)$ \\
\hline $\begin{array}{l}\text { Client should go to the nearest health facility immediately and } \\
\text { inform provider of method use, if there is a sudden change in } \\
\text { health }\end{array}$ & 72.5 & 84.9 \\
\hline $\begin{array}{l}\text { Incorrect } \\
\text { Correct }\end{array}$ & & \\
\hline
\end{tabular}

Table 5: Injectable knowledge retention from posttest to 9 months among CPs and Tier 2 PPMVs $(n=485)$ 
Posttest $(n=485)$

9 months later, among those that answered correctly at posttest

Syringe and needle are used to administer Depo- $\quad(n=420)$

Provera

Incorrect

12.2

7.9

Correct

86.6

92.1

$(n=451)$

Depo-Provera is administered intramuscularly

Incorrect

7.0

15.7

Correct

93.0

84.3

$(n=385)$

Depo-Provera can be administered in buttocks, deltoid, thighs

Incorrect

20.6

19.5

Correct

79.4

80.5

Depo-Provera is administered every 3 months

$(n=460)$

Incorrect

5.2

3.5

Correct

94.8

96.5

Uniject is used to administer Sayana Press

$(n=429)$

Incorrect

8.7

Correct

88.5

$(n=441)$

Sayana Press is administered subcutaneously

Incorrect

9.1

9.3

Correct

90.9

90.7

Sayana Press can be administered on thighs, abdominal wall, back of the arm

Incorrect

44.7

48.1

Correct

53.6

51.9

Sayana Press is administered every 3 months

$(n=416)$

Incorrect

14.2

9.6 


\begin{tabular}{lll}
\hline Posttest $(\mathrm{n}=485)$ & $\begin{array}{l}\text { 9 months later, among those that } \\
\text { answered correctly at posttest }\end{array}$ & \\
\hline Correct & 85.8 & 90.4 \\
\hline $\begin{array}{l}\text { Depo Provera/Sayana Press have same active } \\
\text { drug }\end{array}$ & $(\mathrm{n}=429)$ & 4.2 \\
\hline Incorrect & 11.5 & 95.8 \\
\hline Correct & 88.5 & \\
\hline Sharp box contents disposed at health facility & $(\mathrm{n}=205)$ & 17.6 \\
\hline Incorrect & 57.7 & 82.4 \\
\hline Correct & 42.3 & \\
\hline $\begin{array}{l}\text { Injectables stored at room temperature and out of } \\
\text { direct sunlight }\end{array}$ & $(\mathrm{n}=448)$ & 7.1 \\
\hline Incorrect & 4.7 & 92.9 \\
\hline Correct & 92.4 & \\
\hline
\end{tabular}

Table 6: Implant knowledge retention from posttest to 9 months among CPs and Tier 2 PPMVs $(n=485)$ 
After Jadelle insertion, reusable instruments should be placed in a soapy water, wash and sterilize. (False*)

$$
(n=105)
$$

Incorrect

74.8

92.4

Correct

21.6

7.6

Nexplanon Implant is effective for $\mathbf{3}$ years $(n=399)$

Incorrect

$17.7 \quad 25.3$

Correct

82.3

74.7

Implant is inserted under the skin of the arm $(n=465)$

Incorrect

$\begin{array}{ll}4.1 & 0.4\end{array}$

Correct

95.9

99.6

After Implanon is inserted under the skin, verify the presence of the rod through gentle palpitation

$(n=361)$

Incorrect

21.6

22.7

Correct

74.4

77.3

Implanon NXT needle is inserted at 30 degrees

$(n=225)$

Incorrect

53.6

66.7

Correct

46.4

33.3

Implants inserted into fat under skins may be difficult to remove

$(n=297)$

Incorrect

38.8

33.0

Correct

61.2

67.0

1 st step to implant removal is palpate arm) and mark where the tip of the rod(s) are felt

Incorrect

12.8

24.2

Correct

82.7

75.8

*First step is to decontaminate in chlorine solution before this process.

Table 7. Adjusted odds ratios of FP, injectable and implement knowledge retention 9-months later 


\section{FP knowledge Injectable knowledge Implant knowledge retention $(n=555) \quad$ retention $(n=484) \quad$ retention $(n=484)$}

AOR $\quad 95 \% \mathrm{Cl} \quad$ AOR $\quad 95 \% \mathrm{Cl} \quad$ AOR $\quad 95 \% \mathrm{Cl}$

\section{BCS+ counseling cards, BCS+}

Algorithm or FP flip chart

\begin{tabular}{lllllll} 
Did not receive & ref & \multicolumn{3}{c}{ ref } & \multicolumn{3}{c}{ ref } \\
Received & $2.92^{\star}$ & $\begin{array}{l}1.01- \\
8.40\end{array}$ & 1.01 & $0.32-3.21$ & 1.14 & $0.36-3.60$
\end{tabular}

\section{FP method brochures}

Did not receive

ref

ref

ref

Received

0.85

$0.57-\quad 1.07$

$0.71-1.60$

$0.38^{* *}$

$0.25-0.57$

\section{MEC Wheel}

Did not receive

ref

ref

ref

Received

1.20

$0.63-$

$2.13^{*}$

$1.14-3.992 .24$ *

$1.17-4.28$

2.27

\section{Gender}

Male

ref

ref

ref

Female

1.43

$0.96-$

2.13

$1.74^{\star *}$

$1.17-2.59$

$1.48 *$

$1.0-2.21$

\section{Age}

$21-29$

ref

ref

ref

$30-39$

0.88

$0.50-$

1.55

0.86

$0.48-1.54$

0.91

$0.51-1.62$

$40-49$

0.78

0.43 -

1.43

$50+$

$1.04 \quad 0.54-$

1.15

$0.74-2.71$

0.94

$0.50-1.77$

\section{Cadre}

CP

$5.68^{\star \star}$

$3.25-$
9.92

0.66

$0.37-1.19$

1.90 *

$1.07-3.39$

Tier 2 PPMV

ref

ref

ref

Tier 1 PPMV

$\begin{array}{ll}1.33 & 0.58- \\ & 3.05\end{array}$




\begin{tabular}{lllllll} 
Kaduna & ref & \multicolumn{3}{c}{ ref } & \multicolumn{3}{c}{ ref } \\
Lagos & 0.76 & $0.45-$ & 0.96 & $0.57-1.62$ & $0.38^{\star *}$ & $0.22-0.65$
\end{tabular}

\section{Ever worked in a Health Facility}

\begin{tabular}{lllllll} 
No & ref & \multicolumn{3}{c}{ ref } & \multicolumn{3}{c}{ ref } \\
Yes & $2.22^{\star *}$ & $\begin{array}{l}1.25- \\
3.94\end{array}$ & $2.00^{*}$ & $1.04-3.87$ & 0.88 & $0.45-1.71$
\end{tabular}

\section{Administered injectable in the} last 30 days

\begin{tabular}{lllllll} 
No & - & - & ref & - & - \\
Yes & - & - & 1.48 & $0.94-2.33$ & - & - \\
\hline
\end{tabular}

Administered implant in the last 30 days

$\begin{array}{lllllll}\text { No } & - & - & - & - & \text { ref } & - \\ \text { Yes } & - & - & - & - & 0.89 & 0.54-1.47\end{array}$

${ }^{*} \mathrm{p}$-value $>0.05 ;{ }^{* *} \mathrm{p}$-value $>0.01$ 